\title{
Sub-100 nm Gold Nanomatryoshkas Improve Photo-thermal Therapy Efficacy in Large and Highly Aggressive Triple Negative Breast Tumors
}

\author{
Ciceron Ayala-Orozco ${ }^{\# 1}$, Cordula Urban ${ }^{\# 2}$, Sandra Bishnoi ${ }^{3}$, Alexander Urban ${ }^{3}$, Heather \\ Charron ${ }^{2}$, Tamika Mitchell ${ }^{4}$, Martin Shea ${ }^{4}$, Sarmistha Nanda ${ }^{4}$, Rachel Schiff ${ }^{4}$, Naomi

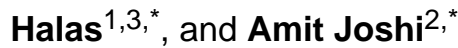 \\ ${ }^{1}$ Department of Chemistry, Rice University, 6100 Main St, Houston, TX 77005, United States \\ 2 Division of Molecular Imaging, Department of Radiology, Baylor College of Medicine, Mail: BCM \\ 360, One Baylor Plaza, Houston, TX 77030, United States \\ ${ }^{3}$ Department of Electrical and Computer Engineering, Rice University, 6100 Main St, Houston, \\ TX 77005, United States \\ ${ }^{4}$ Lester and Sue Smith Breast Center, Baylor College of Medicine, Houston, Texas 77030, \\ United States \\ \# These authors contributed equally to this work.
}

\section{Abstract}

There is an unmet need for efficient near-infrared photothermal transducers for the treatment of highly aggressive cancers and large tumors where the penetration of light can be substantially reduced, and the intra-tumoral nanoparticle transport is restricted due to the presence of hypoxic or nectrotic regions. We report the performance advantages obtained by sub $100 \mathrm{~nm}$ gold nanomatryushkas, comprising of concentric gold-silica-gold layers compared to conventional $\sim 150 \mathrm{~nm}$ silica core gold nanoshells for photothermal therapy of triple negative breast cancer. We demonstrate that a 33\% reduction in silica-core-gold-shell nanoparticle size, while retaining nearinfrared plasmon resonance, and keeping the nanoparticle surface charge constant, results in a four to five fold tumor accumulation of nanoparticles following equal dose of injected gold for both sizes. The survival time of mice bearing large $\left(>1000 \mathrm{~mm}^{3}\right)$ and highly aggressive triple negative breast tumors is doubled for the nanomatryushka treatment group under identical photo-thermal therapy conditions. The higher absorption cross-section of a nanomatryoshka results in a higher efficiency of photonic to thermal energy conversion and coupled with 4-5X accumulation within large tumors results in superior therapy efficacy.

() 2014 Elsevier B.V. All rights reserved.

*Address Correspondence to amitj@bcm.edu, Phone: +1-281-224-9584, MS BCM 360, One Baylor Plaza, Houston, TX 77030. Publisher's Disclaimer: This is a PDF file of an unedited manuscript that has been accepted for publication. As a service to our customers we are providing this early version of the manuscript. The manuscript will undergo copyediting, typesetting, and review of the resulting proof before it is published in its final citable form. Please note that during the production process errors may be discovered which could affect the content, and all legal disclaimers that apply to the journal pertain. 


\section{Keywords}

Nanomatryoshka; Multilayer nanoshells $\mathrm{Au} / \mathrm{SiO}_{2} / \mathrm{Au}$; photothermal therapy; near infrared; gold nanoparticle

\section{INTRODUCTION}

NIR light based nanoparticle-mediated photothermal ablation is a rapidly evolving tool for cancer therapy and compared to conventional treatments including surgery, radiation therapy, and chemotherapy, nanoparticle mediated thermal ablation is minimally invasive, relatively robust with respect to acquired or de novo drug resistance, and offers substantially reduced off-target effects.[1-8] Nanoparticles effective as photothermal transducers are designed to efficiently convert incident light to heat, and ablate malignant cells in nanoparticle vicinity, upon illumination in the favorable NIR region.[9] Gold nanoshells were the first successful NIR photothermal transducers[1], and the NIR guided ablation approaches have been extended to nanoparticles such as gold nanorods[4], gold nanocages[7], hollow gold nanoshells[5], carbon nanotubes[6], and graphene[8], which reproduce NIR plasmonic resonance in a variety of geometries and size regimes.

Gold nanoparticles exhibit NIR activated photothermal activity due to their geometry dependent plasmon resonance, $[1,7,10]$ resulting from collective oscillation of surface electrons upon excitation with light at the resonance frequency. Gold is also attractive because due to its inertness, biocompatibility, low cytotoxicity and long history of medical use. $\sim 150 \mathrm{~nm}$ silica core gold nanoshells are one of the most studied NIR plasmonic photothermal therapy agents with high photothermal conversion efficiency[10], along with non-cytotoxicity and biocompatibility of their component materials, that has led to their approval for initial clinical trials for head and neck cancer treatment.[11] In contrast, $\sim 50 \mathrm{~nm}$ gold nanorods can also offer NIR plasmon resonances; however, they are constrained due to their synthesis procedures which involve high concentrations of cytotoxic cetyltrimethylammonium (CTAB) as a surfactant. CTAB needs to be displaced before therapeutic use and it often results in reshaping and/or irreversible aggregation of nanorods. Nanorod surface functionalization is as a result much more involved than nanoshells.[12-14] Other sub $50 \mathrm{~nm}$ nanostructures based on hollow shell or cage geometries have also been proposed. These agents utilize a sacrifical cobalt or silver core during synthesis, which can raise safety concerns due to leaching of core material,[15] and scalable batch synthesis is difficult.[16, 17],[18] Considering the safety, biocompatibility, and scalable synthesis concerns, silica core gold nanoshells are the mainstay of NIR photothermal therapy but they are constrained by $\sim 150 \mathrm{~nm}$ size requirements for NIR resonance, providing motivation for development of sub $100 \mathrm{~nm}$ silica-gold structures which retain the efficacy and biocompatibility advantages of nanoshells. Similar to gold nanoshells, Gold nanomatryoshkas comprise of silica and gold but with crucial shape differences. Nanomatryoshkas consist of a silica layered Au core, further coated with a thin gold shell. [19] Plasmonic interaction between the Au core and the Au shell, can be exploited by varying the three core-shell radii involved and NIR resonance achieved at smaller length scales than conventional $\mathrm{SiO}_{2} / \mathrm{Au}$ nanoshells. This development of sub-100 nm 
nanomatryoshkas with identical surface functionalization as gold nanoshells is instrumental for enhanced tumor accumulation and much increased photothermal conversion upon NIR excitation.

Breast cancer treatment is an attractive avenue for the application of NIR imaging and therapeutic methods because of relatively shallow tissue depth of both the primary disease and its loco-regional lymph node metastases, coupled with the low NIR absorption and scattering of breast tissue, which enable light propagation upto multiple centimeters.[20] The standard treatment protocol for breast cancer involves surgery accompanied by chemotherapy and/or radiation treatment. Much of the recent progress in cancer therapy has involved the treatment of early stage disease by this approach.[21, 22] However, the past decade has seen a significant change in the statistics of breast cancer diagnosis. Since 2000, the incidence rate of smaller tumors $(\varsigma .0 \mathrm{~cm})$ among women of all races has declined by $3.3 \%$ per year and by $1.1 \%$ per year during 2006-2010, while the incidence rate of larger tumors $(2.1-5 \mathrm{~cm}$ and $>5.0 \mathrm{~cm})$ has remained stable or increased by approximately $0.7-1.1$ $\%$ per year. Larger tumor size at diagnosis is associated with distant metastases and decreased survival, dropping from the 5-year survivability rate of $95 \%$ for tumors $\mathcal{s} \mathrm{cm}$ to $65 \%$ for tumors $>5 \mathrm{~cm}$.[23] Clearly, the standard treatment is significantly less effective for patients with more advanced stages of the disease. Since currently more than $75 \%$ of all breast cancer diagnoses occur at more advanced stages,[23] it is imperative that effective therapeutic strategies for breast cancer beyond early stage disease be developed. In this light, we report efficacy studies on tumors about 10-70 fold larger than the average size used in any previous study of photothermal therapy efficacy assisted by representative classes of nanoparticles (see Table 1). Almost all reported nanoparticle mediated studies are on sub $200 \mathrm{~mm}^{3}$ xenografted tumors, which are typically well vascularized and do not pose the transport barriers expected in advanced disease. We believe that the large tumors are a clinically relevant model to advanced, localized cancers.

Here we report sub-100 nm gold nanomatryoshkas as efficient photothermal transducers for the therapy of large $\left(\sim 1000 \mathrm{~mm}^{3}\right)$ tumors of a highly-aggressive triple negative breast cancer (TNBC) variant. The subtype TNBC is characterized by the lack of estrogen receptor, progesterone receptor, and epidermal growth factor receptor 2 (HER2).[24] The lack of these receptors is associated with high aggressiveness, poor prognosis, shorter survival, and non-response to endocrine therapies and immune therapy against HER2.[24-26] Further, inflammatory breast cancer, which exhibits local recurrence post surgery and radiation therapy is also frequently of the triple negative type. [27]. While fatalities in breast cancer are mostly due to distant organ metastasis, treatment of local disease and local lymph node removal has a high morbidity and treatment related cosmetic challenges, in addition to the chances of developing post surgery lymphedema.[28] Local nanoparticle enabled photothermal therapy can substantially improve the quality of life of breast cancer patients, due to its non-ionizing, repeatable, and externally controlled nature. Active targeting of nanomedicines to TNBC has not been convincingly demonstrated, thus enhanced permeability and retention (EPR) effect is the only means for particle uptake in tumors following systemic delivery, and multiple EPR based diagnostic and therapeutic nanoparticles have been proposed, utilizing a variety of modalities and nanoparticle materials.[29-31] As EPR-based nanoparticle accumulation in tumors is dependent on 
particle size, $[32,33]$ it is essential to obtain sub $100 \mathrm{~nm}$ sizes. In this study, we demonstrate that using sub-100 nm gold nanomatryohskas enhances accumulation in large tumors (>1000 $\mathrm{mm}^{3}$ ) and improves survival time of mice after photothermal treatment relative to the treatment with the standard $\mathrm{SiO}_{2} / \mathrm{Au}$ nanoshells.

\section{MATERIALS AND METHODS}

\section{Nanoshell and Nanomatryushka Synthesis}

Gold nanoshells were synthesized by standard methods developed by our group and described in detail elsewhere.[42, 43] Gold nanomatryoshkas were synthesized by our previously reported method with several improvements.[19] First, the Au colloid (40 nm citrate NanoXact Gold, nanoComposix) was coated with silica doped with 3-aminopropyl triethoxysilane (APTES) by a modified Stöber process. For this reaction the chemicals were added to an Erlenmeyer flask with a ground glass joint under magnetic stirring in the following order: 1) $21 \mathrm{~mL}$ of Au colloid $\left(7.0 \times 10^{10}\right.$ particles $/ \mathrm{mL}$, citrate-capped $40 \mathrm{~nm} \mathrm{Au}$ sphere, NanoComposix), 2) $180 \mathrm{~mL}$ of 200 proof ethanol (Decon Labs), 3) $1.8 \mathrm{~mL}$ of ammonium hydroxide (28-30\%, EMD Chemicals), 4) $36 \mu \mathrm{L}$ of a solution of $10 \%$ tetraethoxysilane (TEOS, SIT7110.2, Gelest) in ethanol and, 5) $36 \mu \mathrm{L}$ of 10\% APTES (SIA0610.1, Gelest) in ethanol. The reaction was capped and stirred $50 \mathrm{~min}$ at room temperature followed by stirring $24 \mathrm{~h}$ at $4 \mathrm{C}$. Then, the solution was dialyzed for $12 \mathrm{~h}$ in 1 gallon of 200 proof ethanol using a dialysis membrane (Spectra/Por 6, MWCO $=10000$, Spectrum Labs) previously washed with MilliQ grade water to remove residual chemicals and then washed with ethanol to remove excess water. The purpose of dialysis is to remove ammonium hydroxide and the remaining free silanes (TEOS and APTES) from the reaction to decrease aggregation of nanoparticles during centrifugation. The solution was cooled to 4 $\mathrm{C}$ and centrifuged $45 \mathrm{~min}$ at $2000 \mathrm{rcf}$ using $50 \mathrm{~mL}$ plastic tubes. The supernatant was removed and the pellet was redispersed by sonication in a total volume of $4 \mathrm{~mL}$ of ethanol. Centrifugation can be repeated when red color is still observed in the supernatant to recoup more particles. The product obtained here are the APTES-doped silica-coated gold colloid.

Next, the synthesis of the seeded precursor consists of the functionalization of APTESdoped silica surface with Duff colloid (1-2 nm) fabricated by the method described in detail elsewere.[42, 44] First, the APTES-doped silica-coated gold colloids are sonicated for 20 $\mathrm{min}$. Then, in a $50 \mathrm{~mL}$ plastic centrifuge tube, $20 \mathrm{~mL}$ of Duff colloid solution was added, followed by rapid, simultaneous addition of $300 \mu \mathrm{L}$ of $1 \mathrm{M} \mathrm{NaCl}$ and $1 \mathrm{~mL}$ of APTES-doped silica-coated gold colloid. The solution was quickly vortexed and sonicated for $30 \mathrm{~min}$. The resulting mixture was incubated 4 days at room temperature and solution was gently shaken and sonicated 20 min once at day. During the incubation the silica layer is reduced in thickness by chemical etching (hydrolysis) and densely covered with Duff colloids. After the incubation, before centrifuging the solutions were sonicated for $20 \mathrm{~min}$, and then centrifuged $45 \mathrm{~min}$ at $700 \mathrm{rcf}$. The supernatant was centrifuged again. The pellets were recovered and redispersed in $800 \mu \mathrm{L}$ of water by sonication $5 \mathrm{~min}$. The solution were centrifuged again for $30 \mathrm{~min}$ at $700 \mathrm{rcf}$ and redispersed in water by sonication $5 \mathrm{~min}$. Centrifugation was repeated but particles were redispersed and combined in a total volume of $\sim 1 \mathrm{~mL}$ of water. This particle is the seeded precursor used for seeded growth of the outer Au shell as described in 
detail elsewhere.[19, 42] This last step uses the same method to synthesize the Au nanoshells.

\section{Functionalization of nanoparticles with thiol-PEG}

Nanoparticle solutions (about $300 \mathrm{~mL}$ of each nanoparticle) were centrifuged, nanoshells at $240 \mathrm{rcf}$ for $30 \mathrm{~min}$ and nanomatryoshkas at $200 \mathrm{rcf}$ for $30 \mathrm{~min}$. Nanoparticles were redispersed in $10 \mathrm{~mL}$ of Milli-Q water and functionalized with a final concentration of 100 $\mu \mathrm{M}$ thiol-PEG (mPEG-SH, MW 10000, Laysan Bio) for $12 \mathrm{~h}$. The nanoparticle solutions were sterilized using a 0.8/0.2 $\mu \mathrm{m}$ pore size syringe filters (PALL Acrodisc PF $32 \mathrm{~mm}$ ). The nanoparticle solutions were centrifuged (nanoshells $280 \mathrm{rcf}$ for $30 \mathrm{~min}$ and nanomatryoshkas $240 \mathrm{rcf}$ for $30 \mathrm{~min}$ ) and re-dispersed in $4 \mathrm{~mL}$ of sterile $1 \mathrm{mM}$ phosphate buffer $\mathrm{pH}$ 7.3. The $\mathrm{Au}$ content was measured in each sample by ICP-MS and Au concentration was adjusted to $1.5 \mathrm{mg}$ of $\mathrm{Au} / \mathrm{mL}$.

\section{Triple Negative Breast Cancer Xenografts}

Luciferase transfected MDA-MB-231LM2 cells first described in Minn et. al. [45],were maintained in DMEM media (Sigma $\left.{ }^{\circledR}\right)$, with $10 \%$ FBS (Gibco®) and 1\% penicillin and streptomycin (Lonza $\left.{ }^{\circledR}\right)$ and incubated in $5 \% \mathrm{CO}_{2}$ at $37 \mathrm{C}$. Cells were routinely maintained by passaging when they became $80 \%$ confluent. Cells were counted and suspended in sterile PBS to the desired cell number concentration $\left(5 \times 10^{7}\right.$ cells $\left./ \mathrm{ml}\right)$ for injection via procedure described in [46]. Briefly, 4-5 week old female athymic nude mice were sourced from Harlan Sprague Dawley. $1 \times 10^{7}$ cells in a total volume of $200 \mu \mathrm{L}$ were injected subcutaneously in the fat pack of the ribcage on one side. Tumor growth was monitored every two days by measurement with a digital caliper and the tumor volume was calculated with the formula: tumor volume $=1 / 2\left(\right.$ length $\mathrm{x}$ width $\left.{ }^{2}\right)$.

\section{Photothermal therapy efficacy experiments}

Tumors were allowed to grow to $\sim 1000 \mathrm{~mm}^{3}$, then $200 \mu \mathrm{L}$ of either PEG conjugated nanomatryoshkas (NM-PEG), PEG conjugated nanoshells (NS-PEG) or a saline solution were injected into the tail vein. Four hours after injection, the mice were anesthetized with isoflurane and the tumor in the treatment groups (with mouse number $n=9$ for NM-PEG, $\mathrm{n}=9$ for NS-PEG, and $\mathrm{n}=5$ for saline solution injections) was treated for five minutes with a $\mathrm{CW}$-diode laser (Diomed 15Plus, Angio Dynamics) emitting $3 \mathrm{~W} / \mathrm{cm}^{2}$ at a wavelength of $808 \mathrm{~nm}$. The beam was expanded $(\sim 1.2 \mathrm{~cm}$ diameter illuminated region) to cover the tumor surface in its entirety and total time duration of treatment was 5 minutes. During treatment a needle based thermocouple probe was inserted in the tumor core and temperature elevations were recorded for each treatment group. Three mice in each group (saline, NM-PEG, and NS-PEG injected) were left untreated as additional controls. Following treatment, mice were regularly monitored by bioluminescence imaging and manual tumor size measurements. When the tumors reached a volume of $2000 \mathrm{~mm}^{3}$ the mice were euthanized via $\mathrm{CO}_{2}$.

\section{Quantitative Bio-distribution Studies}

When tumors reached the same volume $\left(\sim 1000 \mathrm{~mm}^{3}\right)$ as for the photothermal therapy treatment, 18 mice were randomly divided into two groups with 9 mice per group. One 
group was injected with $200 \mu \mathrm{L}$ NM-PEG and the other group was injected with NS-PEG with equal dose of gold ( $300 \mathrm{ug}$ ). Four hours, $24 \mathrm{~h}$ and $72 \mathrm{~h}$ after injection, three mice per group were sacrificed. Brain, heart, lung, liver, spleen, gut, kidney, blood and tumor were collected, the organs were washed in PBS and stored at $-80{ }^{\circ} \mathrm{C}$ until further investigation. For the gold content analysis, the organs were weighted and digested in $\sim 2 \mathrm{~mL}$ of aqua regia. The samples were purified and diluted in $10 \mathrm{~mL} 1 \%$ aqua regia for inductively coupled plasma mass spectrometry (ICP-MS, Perkin Elmer) analysis. The experiments were carried out in three independent runs for statistical analysis.

\section{Histopathology}

Small tumor parts were washed in PBS, held in $10 \%$ buffered formalin for $24 \mathrm{~h}$, washed in PBS ( $3 \times$ for $20 \mathrm{~min}$ ) and then kept in $70 \%$ ethanol. For the staining, the organs were fixed in paraffin blocks and then cut with a microtome. Vasculature was stained with CD34 (lifespan biosciences) and Alexa Fluor $\AA_{594}$ (Molecular Probes ${ }^{\circledR}$, Invirtogen) and the nucleus was stained with DAPI (Vectors). H\&E staining was performed with hematoxylin and eosin from Sigma-Aldrich. Dark field and fluorescence microscopy of tumor sections were conducted with an Olympus BX 41 microscope with an 40× NA0.6 objective and the bright field microscopy was conducted with a Zeis Axioplan 2 microscope.

\section{RESULTS AND DISCUSSION}

\section{Gold nanomatryoshkas and nanoshells size and charge characterization}

Gold nanomatryoshkas were synthesized by modifying a previously published synthesis.[19] A key modification was to dope the silica layer grown on the gold core with 3-aminopropyl triethoxysilane (APTES) during the $\mathrm{SiO}_{2}$ synthesis, which allows binding of gold colloid (1-2 nm in diameter) at the surface of silica layer. These gold colloids act as seeds for the synthesis of the epi-gold layer of the nanomatryoshka. The silica thickness was precisely controlled by a two step process: (i) first grow a $\sim 16 \mathrm{~nm}$ silica layer on the Au core and (ii) then etch the silica layer to $10 \mathrm{~nm}$ by hydrolysis. Silica core/Au shell nanoshells were synthesized by standard methods previously published. Plasmon resonances in both gold nanomatryoshkas and nanoshells are highly tunable to NIR wavelengths by controlling their core and shell dimensions.[19, 43] The extinction spectrum of gold nanoshells and nanomatryoshkas is shown in Figure 1B. Gold nanomatryoshkas show two plasmon modes: 1) alow-energy plasmon subradiant mode at $783 \mathrm{~nm}$ and 2) a high-energy superradiant plasmon mode at $560 \mathrm{~nm}$; these nanomatryoshkas have dimensions $\left[r_{1}, r_{2}, r_{3}\right]=[21,31,44]$ $\mathrm{nm}$. Nanoshells had the average dimensions $\left[\mathrm{r}_{1}, \mathrm{r}_{2}\right]=[62,76] \mathrm{nm}$.obtained from SEM images of over $300 \mathrm{SiO}_{2}$ core particles and 300 nanoshells. Figures $1 \mathrm{C}$ and $1 \mathrm{D}$ depict the TEM images of NM and NS, respectively. Decreasing the shell thickness facilitates an interaction between the inner and outer part of the metallic shell thus shifting the Plasmon resonance in the NIR region of spectrum. [43] The extinction spectrum of gold nanoshells and nanomatryushkas is shown in Figure 1B.

A key parameter for photo-thermal therapy application is the surface charge, which in turn controls the circulation time following systemic injection of nanoparticles.

Nanomatryoshkas and nanoshells were functionalized with thiol-polyethylene glycol (thiol- 
PEG) of molecular weight $10 \mathrm{kDa}$ to render the nanoparticle surface biocompatible and increase the circulation time in the bloodstream of the mice. PEGylation is a well known and popular strategy for increasing the biocompatibility and tailoring the surface characteristics of gold nanoparticles.[47] The hydrodynamic diameter for NM-PEG and NS-PEG was measured to be $137 \mathrm{~nm}$ and $195 \mathrm{~nm}$ respectively. The surface charge for both the particles following PEGylation was $\sim-5 \mathrm{mV}$. A close to neutral surface charge has been reported in literature as contributory to longer circulation time in the bloodstream, better tumor penetration and evasion of the RES.[48, 49]

\section{Bio-distribution of nanomatryoshkas and nanoshells}

Nanomatryoshkas and nanoshells were injected in mice bearing $\sim 1000 \mathrm{~mm}^{3}$ TNBC xenografts. The dose injected for both nanoparticles was $200 \mu \mathrm{L}$ at the concentration of 1.5 $\mathrm{mg} / \mathrm{mL}$ of $\mathrm{Au}$, which is equivalent to $300 \mu \mathrm{g}$ of Au. Using the density of gold $\left(19.3 \mathrm{~g} / \mathrm{cm}^{3}\right)$ we calculated that this dose of $\mathrm{Au}$ is equivalent to $5.7 \times 10^{10}$ nanomatryoshkas with dimensions $\left[\mathrm{r}_{1}, \mathrm{r}_{2}, \mathrm{r}_{3}\right]=[21,31,44] \mathrm{nm}$ and $1.9 \times 10$ nanoshells with dimensions $\left[\mathrm{r}_{1}, \mathrm{r}_{2}\right]=$ $[62,76] \mathrm{nm}$. Mice were sacrificed at $4 \mathrm{~h}, 24 \mathrm{~h}$, and $72 \mathrm{~h}$ post-injection and the gold content in each tumor was quantified by ICP-MS. Gold content in organs are shown in Figure-2. At all time points the gold content in $\mu \mathrm{g}$ of Au per $\mathrm{g}$ of tumor is about twice higher for nanomatryoshkas than for nanoshells. The mass of Au was converted to the number of nanoparticles in the tumor; results are shown in Figure 2D. Considering the physical dimensions of each type of particle and density of gold $\left(19.3 \mathrm{~g} / \mathrm{cm}^{3}\right)$ we calculated that the mass of Au in a single nanomatryoshka is $5.22 \times 10^{-9} \mu \mathrm{g}$ and for a nanoshell is $1.62 \times 10^{-8}$ $\mu \mathrm{g}$. Overall, there is $\sim 4.5-6.5$ times the number of nanomatryoshkas than nanoshells in the tumor at all time points even though we injected an equal mass of gold. These higher accumulation of nanomatryohskas in tumor relative to nanoshells is due to their smaller size, which facilitates better penetration into tissue.

\section{Photothermal therapy efficacy}

Figure-3 reports the therapy efficacy results for the mice assigned to three experimental groups: 1) NM injection plus laser treatment ( $n=9), 2)$ NS injection plus laser treatment $(\mathrm{n}=9), 3)$ saline solution injection plus laser treatment $(\mathrm{n}=5)$. In addition, three mice per group injected with NM, NS, or saline, but not treated with laser were also observed. All the mice received via tail vein injection $200 \mu \mathrm{L}$ PEG conjugated nanomatryoshkas or nanoshells at equal $\mathrm{Au}$ dose $(300 \mu \mathrm{g}$ of $\mathrm{Au})$, which is equivalent to $5.7 \times 10^{10}$ nanomatryoshkas and $1.9 \times 10^{10}$ nanoshells. Laser photothermal ablation of TNBC tumors was conducted $4 \mathrm{~h}$ after injection. Laser power was $3 \mathrm{~W} / \mathrm{cm}^{2}$ for $5 \mathrm{~min}$. The temperature in the tumor was probed while the treatment was conducted. Figure $3 \mathrm{~A}$ shows the average maximum temperature change in the tumor after $5 \mathrm{~min}$ of laser treatment. After $5 \mathrm{~min}$ of laser treatment the average maximum temperature change in tumor was $25.3 \pm 4.5^{\circ} \mathrm{C}$ for "NM+laser" group, $19.2 \pm 2.9$ ${ }^{\circ} \mathrm{C}$ for "NS+laser" group, and $12.7 \pm 3.1^{\circ} \mathrm{C}$ for "saline+laser" group. Considering the average initial temperature before laser treatment was $32.6 \pm 1.3{ }^{\circ} \mathrm{C}$, the maximum average temperature in tumors was $57.9 \pm 4.7^{\circ} \mathrm{C}$ for "NM+laser" group, $51.8 \pm 3.2$ for "NS+laser" group and $45.3 \pm 3.4$ for "saline+laser" group. The temperature differences were statistically significant as reported by ANOVA analysis $(\mathrm{p}<0.05)$ performed with Prism software. (Prism 6 for Mac OS X, GraphPad Software, La Jolla California USA, www.graphpad.com) The 
higher temperature increase in tumor treated with nanomatryohkas is due to both 1) a higher accumulation of nanomatryoshkas and 2) a higher absorption cross-section of nanomatryoshkas than nanoshells. This higher temperature implies that more heat is produced and enables efficient cancer tumor ablation. Tumor sizes were monitored in mice after treatment and mice were euthanized when tumor size reached $\geq 2000 \mathrm{~mm}^{3}$. At time of therapy the average size of tumors was substantially larger $\left(\sim 1000 \mathrm{~mm}^{3}\right)$ than in any other photothermal therapy efficacy experiment reported $\left(\sim 14-100 \mathrm{~mm}^{3}\right)$. [2, 4, 34, 35] These large tumors sizes mimic the tumor sizes commonly found in patients and provide a closer model for the efficacy of photothermal cancer therapy in the clinic.[50-54] The survival curves of mice are presented in Figure 3B for the treatment and control groups. The mean survival time for the non-laser treated saline group was $6.7 \pm 3.2$ days, $7.2 \pm 4.0$ days for the "saline +laser" group, $11.3 \pm 3.8$ days for the "NS+laser" group, and $15.8 \pm 8.6$ days for the "NM +laser" group. The survival for non-laser treated groups was similar or less than the saline +laser group, indicating the local and laser illumination controlled nature of therapy. The survival curves were compared with Log-rank (Mantel-Cox test) and Logrank test for trends, with $\mathrm{p}<0.05$ for both. Analysis was performed with Prism software. (Prism 6 for Mac OS X, GraphPad Software, La Jolla California USA, www.graphpad.com) Our results show that gold nanomatryoshkas improve the efficacy of photothermal therapy compared with the nanoshells.

\section{Time course of tumor viability following treatment}

Imaging of luciferase bioluminescence can non-invasively track and evaluate the tumor response to the photothermal treatment (Figure-4). Mice injected with saline solution and treated with laser did not exhibit any therapeutic effect as the bioluminescence signal increased monotonically with time. In contrast, mice injected with nanoshells or nanomatryoshkas did present a loss of luciferase activity in the area illuminated by the laser. However, certain portions of tumors did not present with complete remission, these often include the irregular tumor edges. Growth of surviving cancer cells at the edge of the tumors causes a recovery of luciferase activity and tumor relapse. Mice treated with nanomatryoshkas in combination with laser exhibit a longer recovery time of luciferase activity than with nanoshells. In addition, the tumor growth was slower after treatment with nanomatryoshkas than nanoshells. This allowed longer survival time and longer periods of bioluminescence imaging for nanomatryoshkas. This effect can be directly related to higher accumulation of NM in tumors compared to NS. It should be noted that we performed only a single treatment with an expanded laser beam as the objective was to strictly compare only the effect of nanoparticle size while maintaining all other parameters constant. Since large tumors have an irregular structure, tailoring beam shape to specific cases can lead to improved therapy but in this case it would have violated the constancy of therapy conditions, which was carefully maintained for all the treatment groups. Multiple treatments or treatments with an interstitial laser fiber can lead to further improvement in therapy efficacy. Further, an interesting observation was that while the Saline+laser treatment tumors did exhibit temperature increases of $\sim 12$ degree $\mathrm{C}$ above baseline (Figure-3A), the luciferase activity didn't decrease post treatment for any mice, indicating that hyperthermia in absence of nanoparticles had no effect on tumor viability. 


\section{Histopathology}

The cancer cell morphology in tumors before and after laser treatment was investigated through fluorescence microscopy with co-localization of gold nanoparticles by dark field microscopy (Figure-5). The cell nucleus is stained in blue with DAPI; cell cytoplasm in red with Alexa Fluor ${ }^{\circledR 594}$ and gold nanoparticles are observed as yellow bright spots due to nanoparticle light scattering. The tumor samples for these studies were collected at the time of mice sacrifice, which was 6-7 days for non-laser treated mice, and 12-18 days for the laser treated mice depending upon the nanoparticle treatment group. After nanoparticle injection without laser treatment very few gold nanomatryoshkas or nanoshells were detectable in the tumors, most likely because the particles were already cleared out of the tumor at the time of mice euthanasia. In contrast, for the laser treated tumors the nanomatryoshkas and nanoshells were clearly detectable in the tissue most likely because thermal ablation causes fixation of gold nanoparticles in the tumor, and the vascular disruption leads to slow clearance of nanoparticles from tumors. Cancer cell morphology was disrupted with the laser treatment in presence of gold nanoparticles, but not in tumors with control saline solution injections. The hematoxylin and eosin staining confirmed cell destruction when the tumor is treated with Au nanoparticles and laser (Figure 5). But cells were morphologically intact in the absence of Au nanoparticles or laser. The continued presence of both NM and NS in tumors for up to 18 days in laser treated samples promises the potential of repeated therapy for ablating the relapsed tumor. H\&E stained images indicated ablated and scarred regions for both NM and NS treated tumors, however, regions with complete absence of tumor cells, and regrowth of mammary fat cells was only observed in NM treated tumors.

\section{CONCLUSIONS}

Herein, we have reported for the first time the differences in tumor accumulation and therapy outcomes in an advanced triple negative breast cancer model with $>1000 \mathrm{~mm}^{3}$ tumors for two spherical gold-based Near-infrared photothermal transducer nanoparticles, where the only parameter varied was size. The elemental composition, nanoparticle surface coating/charge, and gold dose given to mice was kept constant. Gold nanomatryoshkas showed an improved photothermal therapy efficacy of TNBC when compared to gold nanoshells. The tumor uptake of nanomatryoshkas was 4-5 fold the number of nanoshells when both were injected at equal dose of Au mass. This higher tumor uptake is because nanomatryoshkas are smaller $(\sim 100 \mathrm{~nm})$ in diameter than nanoshells $(\sim 150 \mathrm{~nm})$. A key feature of presented study was the use of highly aggressive and $>1000 \mathrm{~mm}^{3}$ tumors at therapy initiation, which better mimics the locally advanced breast cancer in clinic. Nanoparticle based photo-thermal interventions in locally advanced breast cancer have the potential of reducing or even eliminating the tumors prior to surgery and reduce the treatment morbidity, especially for triple negative breast cancer, where targeted molecular therapies are not available. The limitations of the presented study include the restriction of therapy to primary tumor and a single representative TNBC cell line. These studies will gain further relevance in more advanced orthotopic breast cancer models based on patient derived tumor-grafts, and more extensive cell line panels. Clinically, the fatalities in breast cancer are primarily because of distant organ metastasis. NIR laser based photothermal therapy has 
a unique disadvantage in this regard due to the spatial scales in mice. Since the effective light envelope in tissue is of multiple centimeters from the source, it covers almost the entire mouse volume, and the clearance organs such as liver and spleen will also get ablated, resulted in mouse death. This limitation doesn't exist in humans because of large spatial scales. Hence mouse models are not ideal vehicles for photo-thermally treating organ metastasis, irrespective of the cell line or tumor model employed. The thrust of this paper is to compare the impact of nanoparticle size in advanced tumors following systemic delivery, and its downstream effect on photo-thermal therapy efficacy. These results are likely to be applicable for metastasis as well, but nude mouse models may not be the best for that purpose due to treatment related fatalities. Further improvements in therapy outcomes are possible with interstitial laser treatments and percutaneous interventional radiology based methods, which can extend the applications of phototherapy to internal organs in a minimally invasive manner. The development of non-toxic and stable sub $50 \mathrm{~nm}$ NIR resonant gold nanostructures in future can lead to similar improvements in tumor uptake and therapy efficacy over the $100 \mathrm{~nm}$ nanomatryushkas.

\section{Acknowledgments}

This research was supported by the National Science Foundation under grant NSF-MRI-ECCS-1040470, the National Institute of Health for grants NIH-ROI 5R01 CA151962 and NIH UO1 CA151886. Partial support was also provided by Dan L. Duncan Cancer Center Grant15 P30CA125123 and SPORE Grant No. P50 CA58183 from the National Cancer Institute, Breast Cancer Research Foundation (R. Schiff), and Research Grant and Susan G. Komen for the Cure Foundation Promise Grant PG12221410. Dr. Shawn Zhang from Lester and Sue Smith Breast Center graciously shared the MDA-MB-231-LM2 cells with us.

\section{CITATIONS}

1. Hirsch LR, Stafford RJ, Bankson JA, Sershen SR, Rivera B, Price RE, Hazle JD, Halas NJ, West JL. Nanoshell-mediated near-infrared thermal therapy of tumors under magnetic resonance guidance. Proceedings of the National Academy of Sciences of the United States of America. 2003; 100:13549-13554. [PubMed: 14597719]

2. O'Neal DP, Hirsch LR, Halas NJ, Payne JD, West JL. Photo-thermal tumor ablation in mice using near infrared-absorbing nanoparticles. Cancer Lett. 2004; 209:171-176. [PubMed: 15159019]

3. Loo C, Lowery A, Halas N, West J, Drezek R. Immunotargeted Nanoshells for Integrated Cancer Imaging and Therapy. Nano Letters. 2005; 5:709-711. [PubMed: 15826113]

4. Dickerson EB, Dreaden EC, Huang X, El-Sayed IH, Chu H, Pushpanketh S, McDonald JF, ElSayed MA. Gold nanorod assisted near-infrared plasmonic photothermal therapy (PPTT) of squamous cell carcinoma in mice. Cancer Letters. 2008; 269:57-66. [PubMed: 18541363]

5. Melancon MP, Lu W, Yang Z, Zhang R, Cheng Z, Elliot AM, Stafford J, Olson T, Zhang JZ, Li C. In vitro and in vivo targeting of hollow gold nanoshells directed at epidermal growth factor receptor for photothermal ablation therapy. Molecular Cancer Therapeutics. 2008; 7:1730-1739. [PubMed: 18566244]

6. Moon HK, Lee SH, Choi HC. In vivo near-infrared mediated tumor destruction by photothermal effect of carbon nanotubes. ACS Nano. 2009; 3:3707-3713. [PubMed: 19877694]

7. Chen J, Glaus C, Laforest R, Zhang Q, Yang M, Gidding M, Welch MJ, Xia Y. Gold Nanocages as Photothermal Transducers for Cancer Treatment. Small. 2010; 6:811-817. [PubMed: 20225187]

8. Yang K, Zhang S, Zhang G, Sun X, Lee S-T, Liu Z. Graphene in Mice: Ultrahigh In Vivo Tumor Uptake and Efficient Photothermal Therapy. Nano Letters. 2010; 10:3318-3323. [PubMed: 20684528]

9. Weissleder R. A clearer vision for in vivo imaging. Nature biotechnology. 2001; 19:316-317. 
10. Cole JR, Mirin NA, Knight MW, Goodrich GP, Halas NJ. Photothermal Efficiencies of Nanoshells and Nanorods for Clinical Therapeutic Applications. The Journal of Physical Chemistry. 2009; C(113):12090-12094.

11. Gad SC, Sharp KL, Montgomery C, Payne JD, Goodrich GP. Evaluation of the Toxicity of Intravenous Delivery of Auroshell Particles (Gold-Silica Nanoshells). International Journal of Toxicology. 2012; 31:584-594. [PubMed: 23212452]

12. Gentili D, Ori G, Comes Franchini M. Double phase transfer of gold nanorods for surface functionalization and entrapment into PEG-based nanocarriers. Chemical Communications. 2009:5874-5876. [PubMed: 19787126]

13. Leonov AP, Zheng J, Clogston JD, Stern ST, Patri AK, Wei A. Detoxification of gold nanorods by treatment with polystyrenesulfonate. ACS Nano. 2008; 2:2481-2488. [PubMed: 19206282]

14. Vigderman L, Manna P, Zubarev ER. Quantitative replacement of cetyl trimethylammonium bromide by cationic thiol ligands on the surface of gold nanorods and their extremely large uptake by cancer cells. Angewandte Chemie - International Edition. 2012; 51:636-641.

15. Goodman AM, Cao Y, Urban C, Neumann O, Ayala-Orozco C, Knight MW, Joshi A, Nordlander P, Halas NJ. The Surprising in Vivo Instability of Near-IR-Absorbing Hollow Au-Ag Nanoshells. ACS Nano. 2014

16. Kwon YM, Xia Z, Glyn-Jones S, Beard D, Gill HS, Murray DW. Dose-dependent cytotoxicity of clinically relevant cobalt nanoparticles and ions on macrophages in vitro. Biomedical Materials. 2009; 4

17. AshaRani PV, Mun GLK, Hande MP, Valiyaveettil S. Cytotoxicity and genotoxicity of silver nanoparticles in human cells. ACS Nano. 2009; 3:279-290. [PubMed: 19236062]

18. Sun Y, Xia Y. Alloying and Dealloying Processes Involved in the Preparation of Metal Nanoshells through a Galvanic Replacement Reaction. Nano Letters. 2003; 3:1569-1572.

19. Bardhan R, Mukherjee S, Mirin NA, Levit SD, Nordlander P, Halas NJ. Nanosphere in-ananoshell: A simple nanomatryushka. Journal of Physical Chemistry. 2010; C(114):7378-7383.

20. Ntziachristos V, Yodh A, Schnall MD, Chance B. MRI-guided diffuse optical spectroscopy of malignant and benign breast lesions. Neoplasia (New York, NY). 2002; 4:347.

21. A.C. Society. Cancer Facts and Figures 2014. 2014

22. Veronesi U, Goldhirsch A, Boyle P, Orecchia R, Viale G. Breast cancer. Discovery medicine. 2005; 5:271-277. [PubMed: 20704887]

23. A.C. Society. Breast Cancer Facts and Figures 2013-2014. Atlanta: 2013.

24. Dent R, Trudeau M, Pritchard KI, Hanna WM, Kahn HK, Sawka CA, Lickley LA, Rawlinson E, Sun P, Narod SA. Triple-negative breast cancer: Clinical features and patterns of recurrence. Clinical Cancer Research. 2007; 13:4429-4434. [PubMed: 17671126]

25. Bauer KR, Brown M, Cress RD, Parise CA, Caggiano V. Descriptive analysis of estrogen receptor (ER)-negative, progesterone receptor (PR)-negative, and HER2-negative invasive breast cancer, the so-called triple-negative phenotype: A population-based study from the California Cancer Registry. Cancer. 2007; 109:1721-1728. [PubMed: 17387718]

26. Silver DP, Richardson AL, Eklund AC, Wang ZC, Szallasi Z, Li Q, Juul N, Leong CO, Calogrias D, Buraimoh A, Fatima A, Gelman RS, Ryan PD, Tung NM, De Nicolo A, Ganesan S, Miron A, Colin C, Sgroi DC, Ellisen LW, Winer EP, Garber JE. Efficacy of neoadjuvant cisplatin in triplenegative breast cancer. Journal of Clinical Oncology. 2010; 28:1145-1153. [PubMed: 20100965]

27. Masuda H, Baggerly KA, Wang Y, Iwamoto T, Brewer T, Pusztai L, Kai K, Kogawa T, Finetti P, Birnbaum D, Dirix L, Woodward WA, Reuben JM, Krishnamurthy S, Symmans W, Van Laere SJ, Bertucci F, Hortobagyi GN, Ueno NT. Comparison of molecular subtype distribution in triplenegative inflammatory and non-inflammatory breast cancers. Breast cancer research : BCR. 2013; 15:R112. [PubMed: 24274653]

28. Monleon S, Murta-Nascimento C, Bascuas I, Macia F, Duarte E, Belmonte R. Lymphedema Predictor Factors after Breast Cancer Surgery: A Survival Analysis. Lymphatic research and biology. 2014

29. Rai P, Mallidi S, Zheng X, Rahmanzadeh R, Mir Y, Elrington S, Khurshid A, Hasan T. Development and applications of photo-triggered theranostic agents. Advanced drug delivery reviews. 2010; 62:1094-1124. [PubMed: 20858520] 
30. Berlin JM, Leonard AD, Pham TT, Sano D, Marcano DC, Yan S, Fiorentino S, Milas ZL, Kosynkin DV, Price BK. Effective drug delivery, in vitro and in vivo, by carbon-based nanovectors noncovalently loaded with unmodified paclitaxel. ACS nano. 2010; 4:4621-4636. [PubMed: 20681596]

31. Yang L, Peng X-H, Wang YA, Wang X, Cao Z, Ni C, Karna P, Zhang X, Wood WC, Gao X. Receptor-targeted nanoparticles for in vivo imaging of breast cancer. Clinical Cancer Research. 2009; 15:4722-4732. [PubMed: 19584158]

32. Chauhan VP, Stylianopoulos T, Martin JD, PopoviÄ Z, Chen O, Kamoun WS, Bawendi MG, Fukumura D, Jain RK. Normalization of tumour blood vessels improves the delivery of nanomedicines in a size-dependent manner. Nature Nanotechnology. 2012; 7:383-388.

33. Wong C, Stylianopoulos T, Cui J, Martin J, Chauhan VP, Jiang W, Popovíc Z, Jain RK, Bawendi MG, Fukumura D. Multistage nanoparticle delivery system for deep penetration into tumor tissue. Proceedings of the National Academy of Sciences of the United States of America. 2011; 108:2426-2431. [PubMed: 21245339]

34. Day E, Thompson P, Zhang L, Lewinski N, Ahmed N, Drezek R, Blaney S, West J. Nanoshellmediated photothermal therapy improves survival in a murine glioma model. J Neurooncol. 2011; 104:55-63. [PubMed: 21110217]

35. Gobin AM, Watkins EM, Quevedo E, Colvin VL, West JL. Near-Infrared-Resonant Gold/Gold Sulfide Nanoparticles as a Photothermal Cancer Therapeutic Agent. Small. 2010; 6:745-752. [PubMed: 20183810]

36. Goodrich GP, Bao L, Gill-Sharp K, Sang KL, Wang J, Payne JD. Photothermal therapy in a murine colon cancer model using near-infrared absorbing gold nanorods. Journal of Biomedical Optics. 2010; 15:018001-018001. [PubMed: 20210487]

37. Von Maltzahn G, Park JH, Agrawal A, Bandaru NK, Das SK, Sailor MJ, Bhatia SN. Computationally guided photothermal tumor therapy using long-circulating gold nanorod antennas. Cancer Research. 2009; 69:3892-3900. [PubMed: 19366797]

38. Burke A, Ding X, Singh R, Kraft RA, Levi-Polyachenko N, Rylander MN, Szot C, Buchanan C, Whitney J, Fisher J, Hatcher HC, D'Agostino R Jr, Kock ND, Ajayan PM, Carroll DL, Akman S, Torti FM, Torti SV. Long-term survival following a single treatment of kidney tumors with multiwalled carbon nanotubes and near-infrared radiation. Proceedings of the National Academy of Sciences of the United States of America. 2009; 106:12897-12902. [PubMed: 19620717]

39. Liu X, Tao H, Yang K, Zhang S, Lee ST, Liu Z. Optimization of surface chemistry on singlewalled carbon nanotubes for in vivo photothermal ablation of tumors. Biomaterials. $2011 ; 32: 144$ 151. [PubMed: 20888630]

40. Yang K, Hu L, Ma X, Ye S, Cheng L, Shi X, Li C, Li Y, Liu Z. Multimodal imaging guided photothermal therapy using functionalized graphene nanosheets anchored with magnetic nanoparticles. Advanced Materials. 2012; 24:1868-1872. [PubMed: 22378564]

41. Yang K, Wan J, Zhang S, Tian B, Zhang Y, Liu Z. The influence of surface chemistry and size of nanoscale graphene oxide on photothermal therapy of cancer using ultra-low laser power. Biomaterials. 2012; 33:2206-2214. [PubMed: 22169821]

42. Brinson BE, Lassiter JB, Levin CS, Bardhan R, Mirin N, Halas NJ. Nanoshells Made Easy: Improving Au Layer Growth on Nanoparticle Surfaces. Langmuir. 2008; 24:14166-14171. [PubMed: 19360963]

43. Oldenburg SJ, Averitt RD, Westcott SL, Halas NJ. Nanoengineering of optical resonances. Chemical Physics Letters. 1998; 288:243-247.

44. Duff DG, Baiker A, Edwards PP. A new hydrosol of gold clusters. 1. Formation and particle size variation. Langmuir. 1993; 9:2301-2309.

45. Minn AJ, Gupta GP, Siegel PM, Bos PD, Shu W, Giri DD, Viale A, Olshen AB, Gerald WL, Massague J. Genes that mediate breast cancer metastasis to lung. Nature. 2005; 436:518-524. [PubMed: 16049480]

46. Rimawi MF, Wiechmann LS, Wang YC, Huang C, Migliaccio I, Wu MF, Gutierrez C, Hilsenbeck SG, Arpino G, Massarweh S, Ward R, Soliz R, Osborne CK, Schiff R. Reduced dose and intermittent treatment with lapatinib and trastuzumab for potent blockade of the HER pathway in 
HER2/neu-overexpressing breast tumor xenografts. Clinical cancer research : an official journal of the American Association for Cancer Research. 2011; 17:1351-1361. [PubMed: 21138857]

47. Shenoy D, Fu W, Li J, Crasto C, Jones G, DiMarzio C, Sridhar S, Amiji M. Surface functionalization of gold nanoparticles using hetero-bifunctional poly (ethylene glycol) spacer for intracellular tracking and delivery. International journal of nanomedicine. 2006; 1:51. [PubMed: 16467923]

48. Arvizo RR, Miranda OR, Moyano DF, Walden CA, Giri K, Bhattacharya R, Robertson JD, Rotello VM, Reid JM, Mukherjee P. Modulating pharmacokinetics, tumor uptake and biodistribution by engineered nanoparticles. PLoS ONE. 2011; 6

49. Lipka J, Semmler-Behnke M, Sperling RA, Wenk A, Takenaka S, Schleh C, Kissel T, Parak WJ, Kreyling WG. Biodistribution of PEG-modified gold nanoparticles following intratracheal instillation and intravenous injection. Biomaterials. 2010; 31:6574-6581. [PubMed: 20542560]

50. Elkin EB, Hudis C, Begg CB, Schrag D. The effect of changes in tumor size on breast carcinoma survival in the U.S.: 1975-1999. Cancer. 2005; 104:1149-1157. [PubMed: 16088887]

51. Carter CL, Allen C, Henson DE. Relation of tumor size, lymph node status, and survival in 24,740 breast cancer cases. Cancer. 1989; 63:181-187. [PubMed: 2910416]

52. Port JL, Kent MS, Korst RJ, Libby D, Pasmantier M, Altorki NK. TUmor size predicts survival within stage ia non-small cell lung cancer. CHEST Journal. 2003; 124:1828-1833.

53. Cheng L, Neumann RM, Scherer BG, Weaver AL, Leibovich BC, Nehra A, Zincke H, Bostwick DG. Tumor size predicts the survival of patients with pathologic stage $\mathrm{t} 2$ bladder carcinoma. Cancer. 1999; 85:2638-2647. [PubMed: 10375113]

54. Hafez KS, Fergany AF, Novick AC. NEPHRON SPARING SURGERY FOR LOCALIZED RENAL CELL CARCINOMA: IMPACT OF TUMOR SIZE ON PATIENT SURVIVAL, TUMOR RECURRENCE AND TNM STAGING. The Journal of Urology. 1999; 162:1930-1933. [PubMed: 10569540] 
A

\section{C}
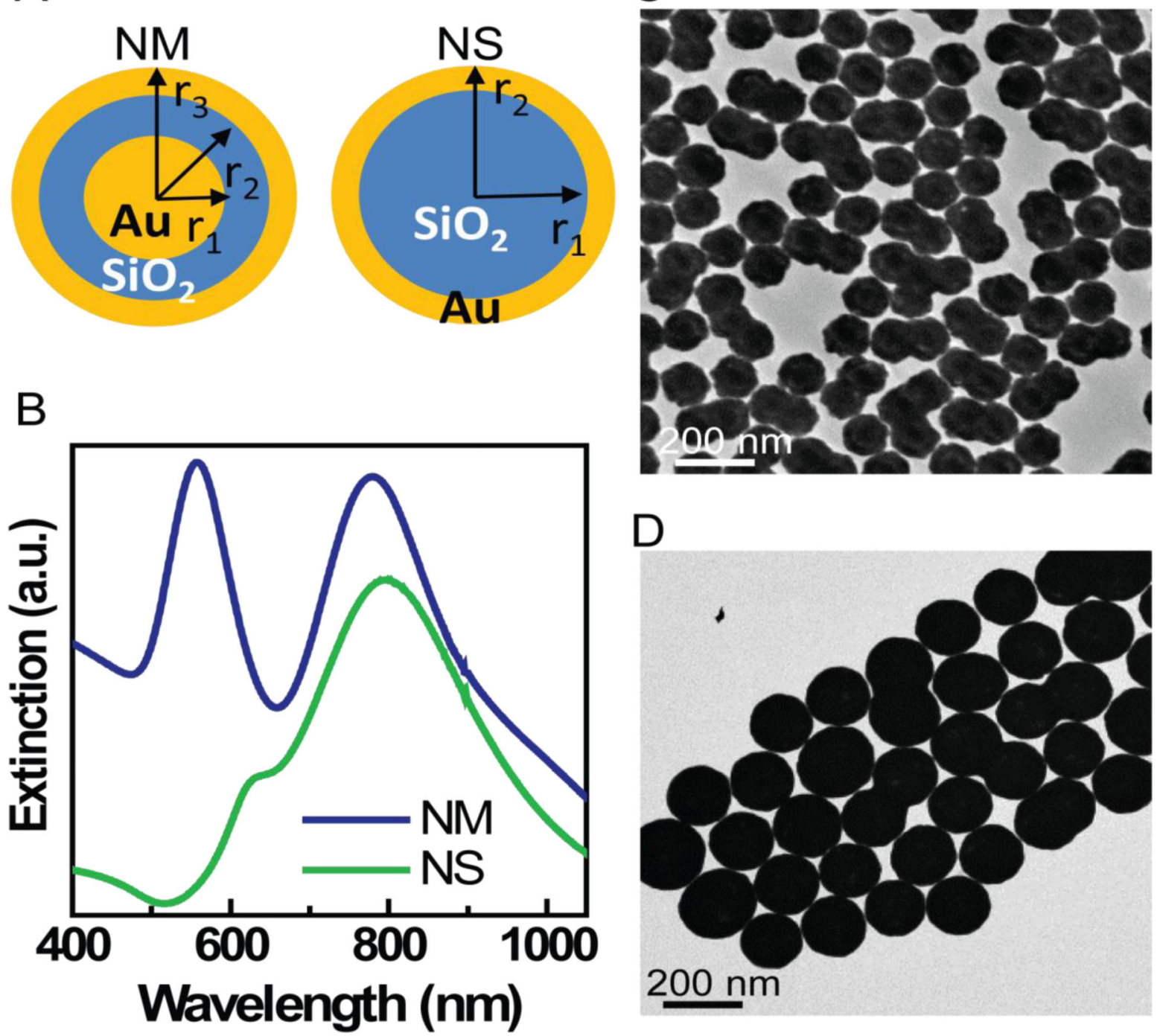

$\mathrm{D}$

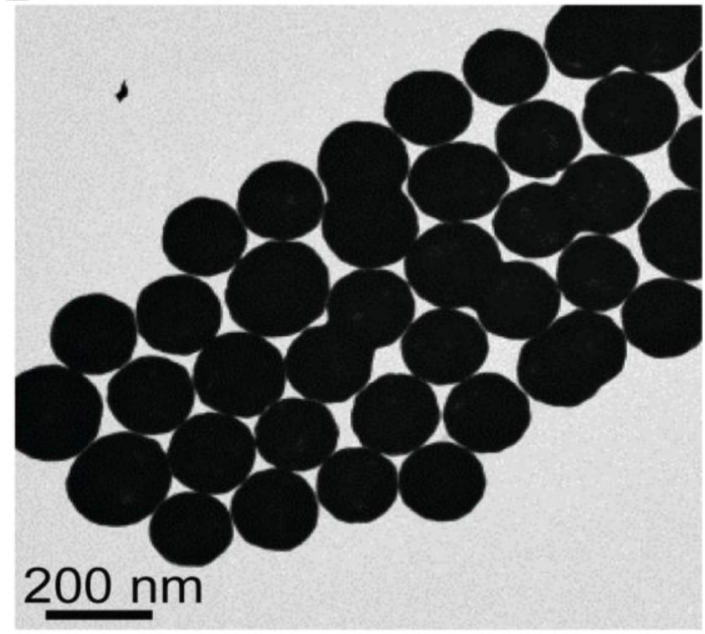

Figure 1.

A. Schematic illustrating the structural differences between nanomatryushka ([r1, r2, r3] = $[21,31,44] \mathrm{nm})$ and nanoshells $([\mathrm{r} 1, \mathrm{r} 2]=[62,76] \mathrm{nm})$, B. Extinction spectra (a.u.) for NM and NS. C. TEM images of NM, and D. TEM images of NS. 
A

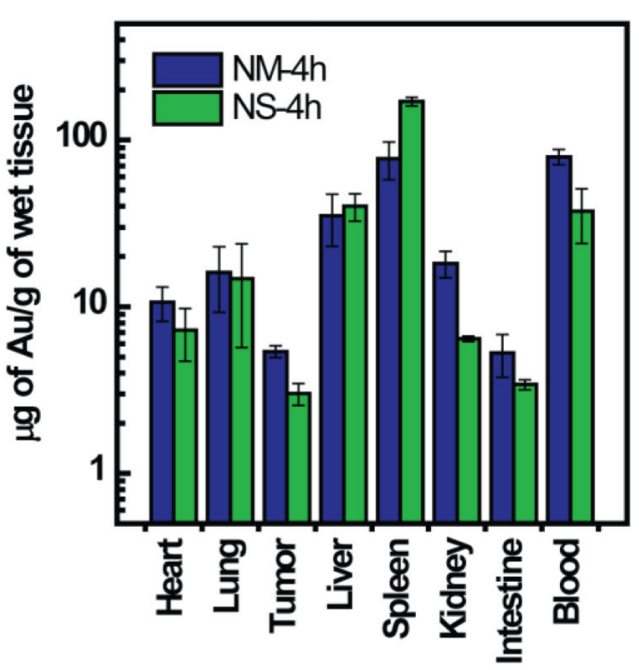

C

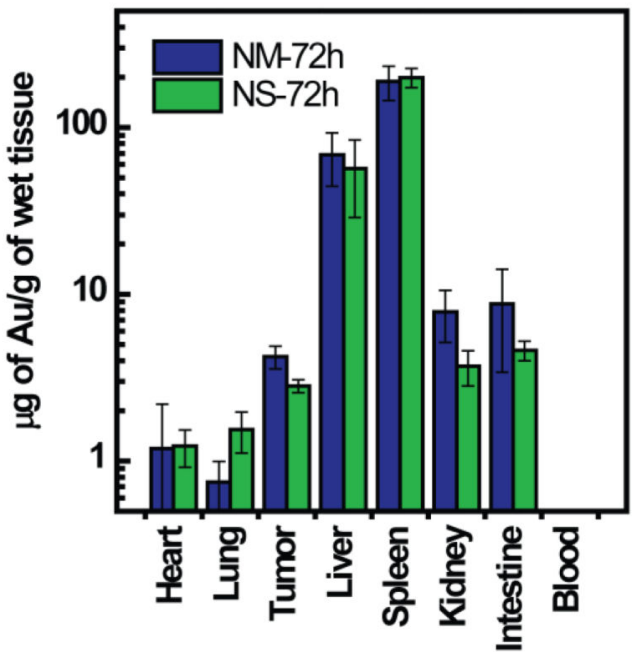

B

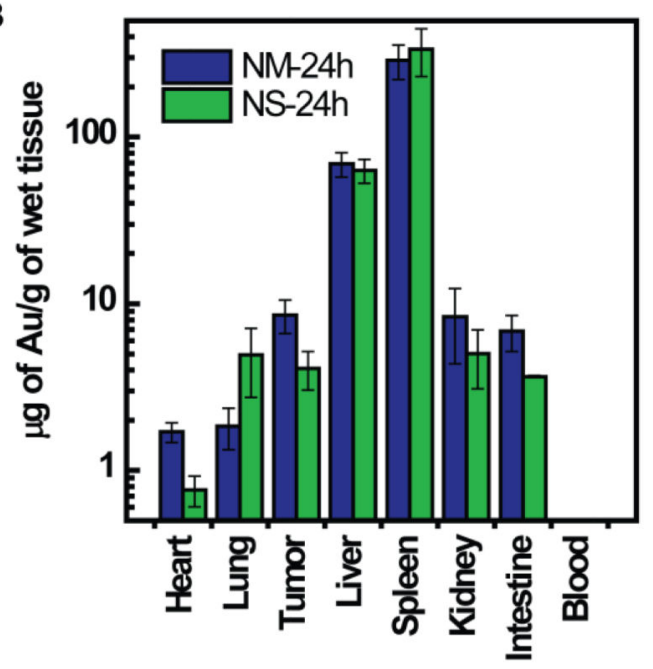

D

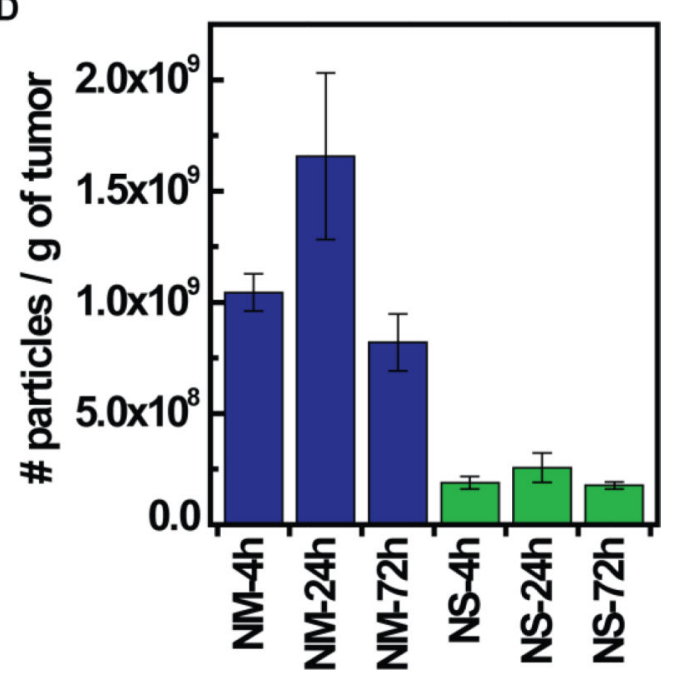

Figure 2.

Bio-distribution of gold NM and NS in mice with large tumors ( $<1000 \mathrm{~mm} 3)$. A) Mean gold content in organs after $4 \mathrm{~h}$ intravenous injection. B) Mean gold content in organs after $24 \mathrm{~h}$ intravenous injection and C) Mean gold content in organs after $72 \mathrm{~h}$ intravenous injection.

D) Mean number of gold nanoparticles in the tumor per gram of wet tissue. Gold concentrations were analyzed by ICP-MS. The number of nanoparticles was calculated considering the mass of Au in a NM $=5.22 \times 10-9 \mu \mathrm{g}$ for NM dimensions $\left[\mathrm{r}_{1}, \mathrm{r}_{2}, \mathrm{r}_{3}\right]=[21$, $31,44] \mathrm{nm}$ and for NS $=1.62 \times 10-8 \mu \mathrm{g}$ of Au for NS with dimensions $\left[\mathrm{r}_{1}, \mathrm{r}_{2}\right]=[62,76] \mathrm{nm}$. The gold content in tumors in $\mu \mathrm{g}$ of $\mathrm{Au} / \mathrm{g}$ of wet tissue is plotted as $\log 10$ scale for visual clarity of values in organs with low gold content. Error bars are drawn with standard error of mean. 

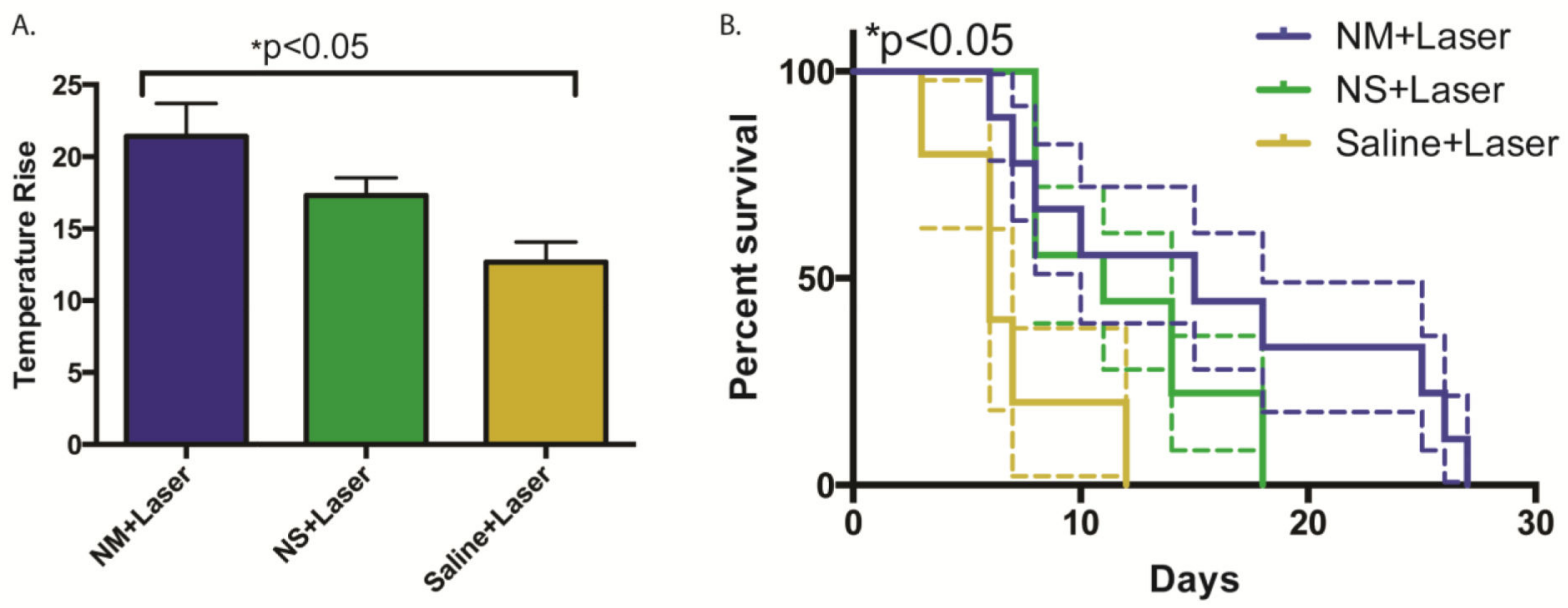

Figure 3.

A) Mean temperature change in treated tumors under 5 min irradiation with a laser of wavelength at $808 \mathrm{~nm}$ and power of $3 \mathrm{~W} / \mathrm{cm}^{2}$, with error bars drawn with standard error B) Survival curves of tumor-bearing mice after photothermal therapy. The average survival times were $6.7 \pm 3.2$ days (for the "saline" group), $7.2 \pm 4.0$ days (for the "saline+laser" group, $11.3 \pm 3.8$ days (for the "NS+laser \pm group), and $15.8 \pm 8.6$ days (for the "NM+laser" group). Standard error bounds are drawn with dashed lines. The results are statistically significant $(\mathrm{p}<0.05)$ 


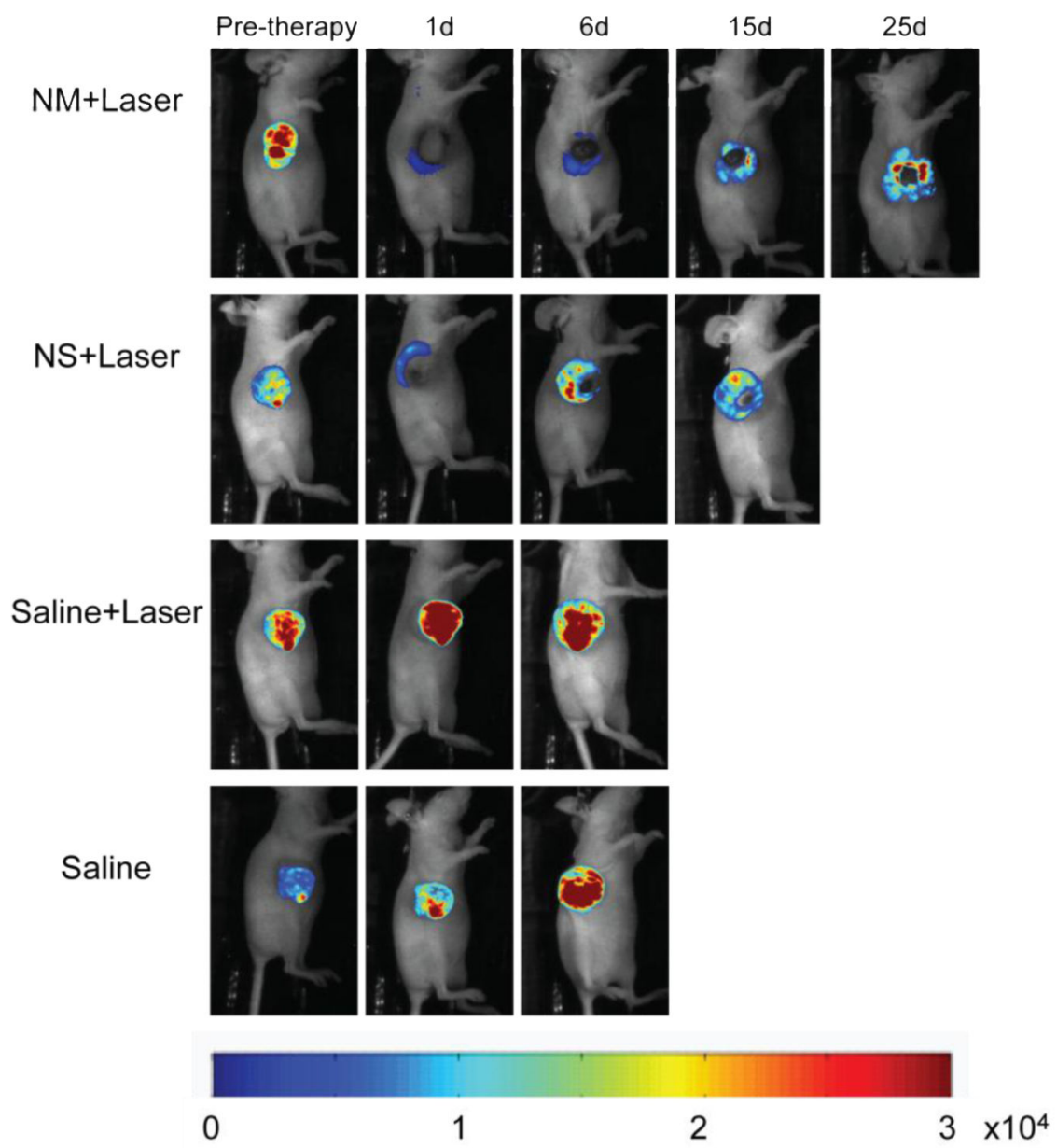

Figure 4.

Evaluation of tumor response to photo-thermal therapy by bioluminescence imaging. The bioluminescence signal is generated only in living cancer cells as result of luciferase activity. The mice treated with laser and nanomatryoshka (NM) or nanoshells (NS) experienced loss of bioluminescence in the area illuminated by the laser spot as seen 1 day after therapy. However, there were viable cancer cells in the edges of the tumor post therapy, which cause tumor recurrence for the highly aggressive cell lines used in this experiment. Mice were euthanized when tumor volume reached $2000 \mathrm{~mm}^{3}$. 
A

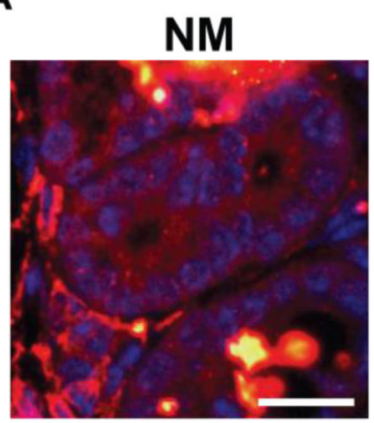

NS

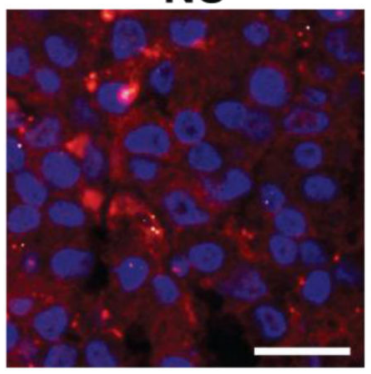

Saline

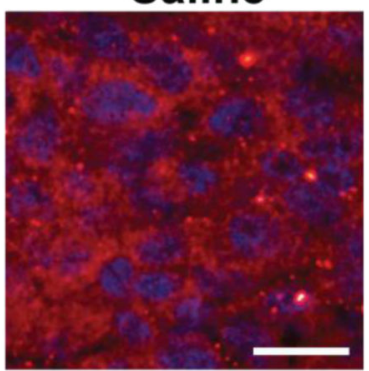

NM+Laser

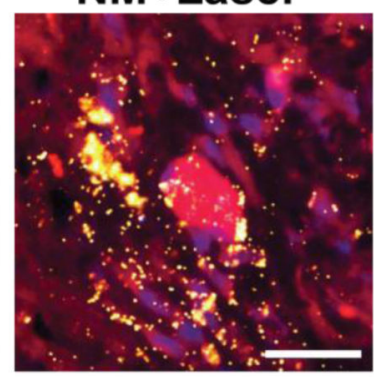

NS+Laser

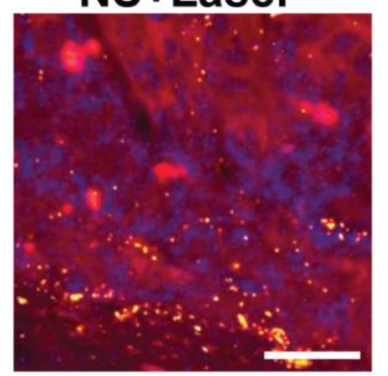

Saline+Laser

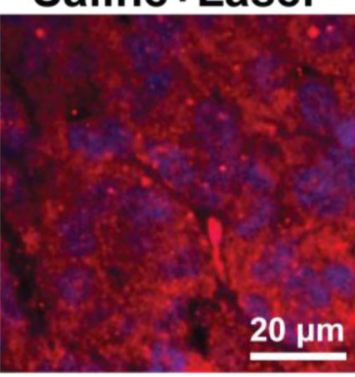

B

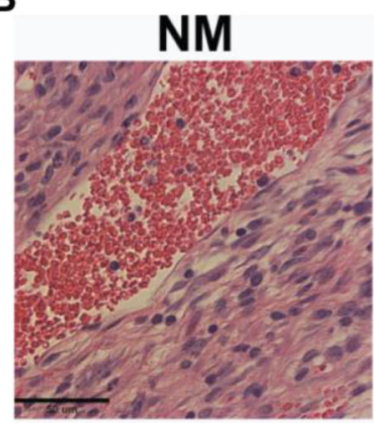

NS

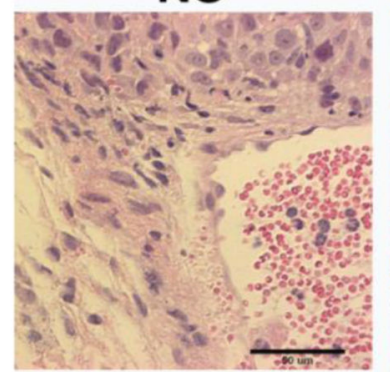

Saline

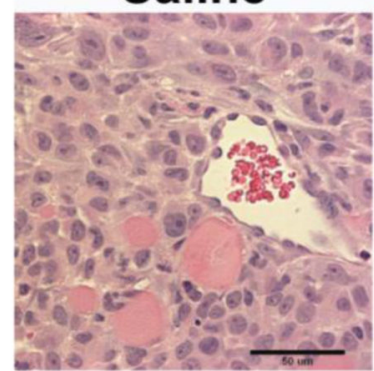

NM+Laser

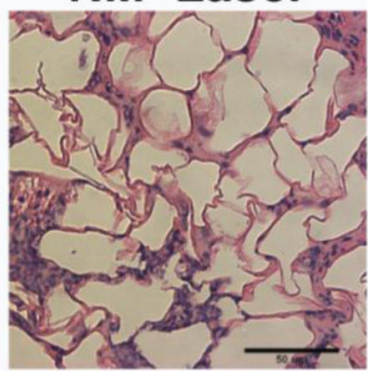

NS+Laser

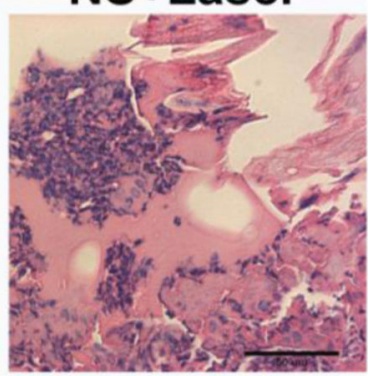

Saline+Laser

Figure 5.

Histopathology of tumor sections extracted from mice intravenously injected with gold nanoparticles or control saline solution. (Left panel) Fluorescence staining combined with dark field microscopy: cell nucleus is stained with DAPI in blue; cell cytoplasm stained with Alexa Fluor ${ }^{\circledR} 594$ in red and gold nanoparticle can be observed as yellow bright spots due to the nanoparticle scattering in the dark field mode. (Right panel) Hematoxyline and Eosine (H\&E) staining. The cell morphology in all non-treated tumors, specially the nucleus, is observed intact. However, after laser treatment in presence of gold nanomatryoshkas or nanoshells the cell morphology is disrupted. 


\section{Table I}

Average tumor volume and diameter used in this study compared with previous photothermal therapy efficacy reports for representative photo-thermal transducers.

\begin{tabular}{|l|l|l|l|}
\hline Volume $\left(\mathbf{m m}^{\mathbf{3}}\right)$ & Diameter $(\mathbf{m m})$ & Nanoparticle type & Reference \\
\hline 1000 & $11.5-15$ & Nanomatryoshka & This study \\
\hline $14-83$ & $3-5.5$ & Nanoshells & {$[2,34,35]$} \\
\hline $14-100$ & $3-6$ & Nanorods & {$[4,36,37]$} \\
\hline 83 & 5.5 & Carbon nanotubes & {$[6,38,39]$} \\
\hline $50-100$ & $4.5-6$ & Graphene & {$[8,40,41]$} \\
\hline
\end{tabular}

